\title{
Reactivity of Aluminum Lactate-Modified Nanoscale Iron Particles with Pentachlorophenol in Soils
}

\author{
Kenneth Darko-Kagya, Amid P. Khodadoust, ${ }^{*}$ and Krishna R. Reddy \\ Department of Civil and Materials Engineering, University of Illinois at Chicago, Chicago, Illinois.
}

Received: March 13, $2010 \quad$ Accepted in revised form: July 13, 2010

\begin{abstract}
Due to the hindered transport of nanoscale iron particles (NIP) in the subsurface caused by the agglomeration and adsorption of NIP during transport, the NIP surface has to be modified to improve the transport of NIP in the subsurface. This study assessed the effectiveness of surface-modified NIP for in situ degradation of contaminants using aluminum lactate as the modifying agent, where aluminum lactate had been earlier shown to be an effective dispersant for enhanced transport of modified NIP in the subsurface. The reactivity of bare-NIP and lactate-modified NIP (LM-NIP) was investigated using pentachlorophenol (PCP) as a representative chlorinated hydrophobic organic contaminant in kaolin and field sand as low permeability clayey soil and high permeability soil, respectively. Kaolin and field sand were spiked at $100 \mathrm{mg} / \mathrm{kg}$ PCP and at $89 \mathrm{mg} / \mathrm{kg}$ PCP, respectively. NIP dosages of $4,10,20,50,75$, and $100 \mathrm{~g} / \mathrm{L}$ were applied for a reaction period of $24 \mathrm{~h}$. For the bare-NIP dosage of $100 \mathrm{~g} / \mathrm{L}, \mathrm{PCP}$ degradation of $98 \%$ and $99 \%$ was obtained in $24 \mathrm{~h}$ for field sand and kaolin, respectively. The effect of reaction time on reactivity of bare-NIP and LM-NIP was evaluated using a lower NIP dosage of $4 \mathrm{~g} / \mathrm{L}$ for 1, 2, 4,7 , and 14 days. After 14 days, greater PCP degradation occurred in bare-NIP systems than in LM-NIP systems, where $95 \%$ and $99 \%$ degradation was obtained for field sand and kaolin with bare-NIP, respectively, whereas $90 \%$ and $91 \%$ degradation was obtained for field sand and kaolin with LM-NIP, respectively. Differences in PCP degradation between bare-NIP and LM-NIP diminished with time more rapidly for field sand than for kaolin. For both field sand and kaolin, nearly complete dechlorination of PCP to phenol occurred with bare-NIP with a more rapid dechlorination in field sand than in kaolin.
\end{abstract}

Key words: pentachlorophenol; nanoscale iron particles; aluminum lactate; kaolin; field sand; reactivity

\section{Introduction}

$\mathbf{M}$ ANY SITES AROUND the globe are contaminated with chlorinated organic compounds such as pentachlorophenol (PCP), trichloroethylene, and trichloroethane. The contaminants can reside in the groundwater, surface water, or soils. PCP has been used extensively as a general biocide for a variety of purposes such as agriculture and timber preservation. Worldwide use of PCP has led to severe contamination problems particularly around former timber treatment plant sites. PCP was widely used as a wood preservative in the United States for several decades, and currently there are several Superfund sites (surface and subsurface soils) contaminated with PCP, which is considered a priority pollutant by the U.S. Environmental Protection Agency (USEPA) (Keith and Telliard, 1979). Various technologies for remediation of

*Corresponding author: Department of Civil and Materials Engineering, University of Illinois at Chicago, 842 West Taylor St., Chicago, IL 60607. Phone: (312) 996-3435; Fax: (312) 996-2426; E-mail: akhodado@uic.edu
PCP-contaminated soils include soil washing, chemical oxidation, and bioremediation; however, these methods are either ineffective or expensive for subsurface soils.

Nanotechnology has the potential to clean up PCPcontaminated soils and groundwater both in situ and $e x$ situ. Nanotechnology for environmental remediation consists of the use of nanoscale zero-valent iron particles to react with organic and inorganic contaminants. The small size of the particles and a vast surface to volume ratio makes them more reactive and versatile. This is applicable to a wide variety of contaminants. The reactivity of organic compounds with nanoscale iron particles (NIP) is dependant on the dosage of NIP, initial concentration of compounds, and reaction time (Choe et al., 2001; Okinaka et al., 2005; Yang and Lee, 2005). Wang and Zhang (1997) indicated that NIP are able to remediate organic compounds faster than microscale zerovalent iron particles. Tratnyek and Johnson (2006) reported that iron nanoparticles possess high reactivity due to their high surface area, greater density of reactive sites, and higher intrinsic reactivity on reactive sites. The utility of zero-valent iron as an effective reductant for treatment of contaminants is due to the generation of several types of reducing species 
during iron corrosion. The standard reductive potential of zero-valent iron is $447 \mathrm{mV}\left[\mathrm{Fe} \rightarrow \mathrm{Fe}^{2+}+2 \mathrm{e}^{-} ; E_{\mathrm{h}}{ }^{0}=447 \mathrm{mV}\right]$. Among possible reduction reactions involving iron and a halogenated organic compound, RX, dissolved in the aqueous phase, the predominant reaction between dissolved RX (e.g., $\mathrm{PCP})$ and zero-valent iron is the heterogeneous reaction occurring at the surface of the metal rather than the reactions with hydrogen and ferrous iron in the aqueous phase:

$$
\mathrm{RX}+\mathrm{Fe}+\mathrm{H}^{+}=\mathrm{RH}+\mathrm{Fe}^{2+}+\mathrm{X}^{-}
$$

Kim and Carraway (2000) found microscale zero-valent iron to be more efficient than other modified zero-valent iron used in their study for dechlorination of PCP, where nearly $50 \%$ of the PCP was removed in a few hours. Morales et al. (2002) showed the affinity of microscale zero-valent iron for the dehalogenation of chlorinated phenols. Cheng et al. (2006, 2008) showed that different isomers of chlorophenol (2-CP, 3$\mathrm{CP}$, and 4-CP) were dechlorinated by NIP and removal increased from $4-\mathrm{CP}$ to $2-\mathrm{CP}$. The percent removal of $4-\mathrm{CP}$, $2-\mathrm{CP}$, and $3-\mathrm{CP}$ was, respectively, $25.6 \%, 32.0 \%$, and $39.9 \%$, where the position of the halogens did have an effect on its removal rate. In addition, temperature affected the rate of removal and reaction pathway as dechlorination was predominant at higher temperature $\left(30^{\circ} \mathrm{C}\right)$, whereas adsorption was the leading process at low temperature of $10^{\circ} \mathrm{C}$. The presence of dissolved oxygen can affect the reactivity of NIP with the target contaminants (Tratnyek and Johnson, 2006). The effect of ionic strength on the reactivity of NIP is minimal at high concentration of NIP (Okinaka et al., 2005). Factors such as $\mathrm{pH}$, redox potential, temperature, anaerobic or aerobic conditions, type of organic compound, dispersed NIP, and dosage of NIP affect the reaction rate.

There have been limited studies on the reactivity of NIP with organic contaminants in soils. NIP can be injected directly into the soil to react with the contaminant in situ. For example, $90 \%$ of PCBs in aqueous solution was degraded as compared with $38 \%$ of PCBs, which were degraded in soils and the difference in reaction rate was due to the difficulty of the PCB in diffusing from the surface of the soil particles to the nanoscale iron surface for effective reaction (Varanasi et al., 2007). Reaction rate in soils also increases with increasing iron concentration and with increasing reaction time and reaction temperature (Varanasi et al., 2007). The potential of reaction with organic contaminants in soils is better for NIP than for microscale iron.

\section{Surface-modified NIP}

Due to agglomeration of the bare-NIP they have limited transport in soil, making it difficult to distribute into the source zone. Bare-NIP is not well dispersed, but modified NIP is more dispersed and homogeneous, and therefore a strong contender for in situ remediation (Kanel and Choi, 2007; Saleh et al., 2007; Reddy et al., 2008).

To directly assess the enhanced transport of NIP in soils, we conducted a systematic study to evaluate the effectiveness of different modifiers or dispersants (Cameselle et al., 2008). Eight different dispersants (three types of lactate, two polymers, and three cyclodextrins) at different concentrations were tested. The dispersants tested were aluminum lactate, sodium lactate, ethyl lactate, polyacrylic acid, aspartic acid, methyl $\beta$-cyclodextrin, beta-cyclodextrin, and hydroxyl propyl-beta- cyclodextrin. The zeta potential of NIP with various surface modifying agents was measured, showing that the zeta potential of bare-NIP was $41.7 \pm 2.3 \mathrm{mV}$. The influence of the dispersants was found to vary depending on the chemical nature of the dispersant and the electrical charge of the ions in solution. Aluminum lactate released $\mathrm{Al}^{3+}$ into the solution, resulting in a reduction of the modified NIP zeta potential from $37.7 \pm 1.8$ to $9.5 \pm 0.7 \mathrm{mV}$ for NIP modified with $2 \%$ and $15 \%$ aluminum lactate, respectively.

Several soil column experiments using NIP modified with the above-mentioned dispersants were conducted using field sand as the porous media. The study showed that $10 \%$ aluminum lactate-modified NIP (LM-NIP) exhibited the highest (93\%) elution of the modified NIP from the field sand. On the basis of the results from column experiments and zeta potential measurements, $10 \%$ aluminum lactate was selected as suitable dispersant for NIP to enhance transport in soils. Lactate is considered a green compound (environmentally benign) and is relatively inexpensive (Subramanian et al., 2010). Moreover, it may enhance the bioremediation of contaminants in soils, making it a favorable alternative for longterm residual treatment if relied on biodegradation.

In this study the degradation of PCP was investigated using bare-NIP and aluminum LM-NIP. The effects of NIP dosage, reaction period, soil type, and aluminum lactate on reactivity of NIP with PCP were examined.

\section{Materials and Methods}

\section{Characteristics of NIP}

The NIP used in this study was obtained from Toda Kogyo. The particles had an average diameter of $70 \mathrm{~nm}$ (with a range of 50-300 nm), $\mathrm{pH}$ of 10.7 , and BET surface area of $37.1 \mathrm{~m}^{2} / \mathrm{g}$. On the basis of X-ray diffraction, the NIP are found to consist of an elemental iron core $(\alpha-\mathrm{Fe})$ and a magnetite shell $\left(\mathrm{Fe}_{3} \mathrm{O}_{4}\right)$ with $\sim 50 \mathrm{wt} \% \quad \alpha$-Fe core and $50 \mathrm{wt} \% \mathrm{Fe}_{3} \mathrm{O}_{4}$. The average particle size determined with a scanning electron microscope is $70 \mathrm{~nm}$. The density of the aqueous NIP particle suspension is $1.27 \mathrm{~g} / \mathrm{mL}$ at solids concentration of $25.6 \mathrm{wt} \%$. The sulfur content is $\sim 4,500 \mathrm{mg} / \mathrm{kg}$ and it originates from the ferrous sulfate starting material used for the production of NIP. These particles are manufactured in bulk and available presently at a cost of \$25 to $\$ 30$ per pound. Because of the characteristics of NIP as relatively environmentally benign, NIP have been deemed suitable for subsurface contaminant remediation. The main properties of these are summarized in Table 1. These

Table 1. Properties of Nanoscale Iron Particle

\begin{tabular}{ll}
\hline Coercive force $(\mathrm{Hc})$ & $408 \mathrm{Oe}$ \\
Mass magnetization $(\sigma \mathrm{s})$ & $149.6 \mathrm{emu} / \mathrm{g}$ \\
$\sigma \rho / \sigma \mathrm{s}$ (ratio of ferromagnetism and & 0.152 \\
$\quad$ antiferromagnetism) & \\
$\mathrm{pH} \quad$ & 10.7 \\
Surface area (BET) & $37.1 \mathrm{~m}^{2} / \mathrm{g}$ \\
Electrical conductivity & $2.29 \times 10^{2} \mu \mathrm{S} / \mathrm{cm}$ \\
Particle size & $50-300 \mathrm{~nm}$ \\
Aqueous suspension & $20-30 \mathrm{wt} \%$ \\
Density of aqueous slurry & $1.2-1.3 \mathrm{~g} / \mathrm{mL}$ \\
\hline
\end{tabular}

Data provided by TODA Kogyo (Japan). 
NIP possess electromagnetic properties (Liu et al., 2005; Okinaka et al., 2005).

\section{Chemicals}

PCP was used to spike the soils. PCP [MW=266.5] was obtained from Aldrich Chemicals. PCP $\left[\mathrm{C}_{6} \mathrm{Cl}_{5} \mathrm{OH}\right]$ is a hydrophobic organic compound with a $\log K_{\mathrm{ow}}$ of 5.0 and an aqueous solubility of $14 \mathrm{mg} / \mathrm{L}$ at $25^{\circ} \mathrm{C}$. The aluminum lactate $\left[\mathrm{C}_{9} \mathrm{H}_{15} \mathrm{AlO}_{9} ; \mathrm{MW}=294\right]$ used for surface modification of NIP was obtained from Aldrich Chemicals. A simulated groundwater solution was prepared in the laboratory using deionized water; this solution contained sodium bicarbonate $(0.006 \mathrm{M})$, calcium chloride $(0.002 \mathrm{M})$, and magnesium chloride $(0.001 \mathrm{M})$. The $\mathrm{pH}$, total dissolved solids, and electrical conductivity of the simulated groundwater solution were 7.8, $500 \mathrm{mg} / \mathrm{L}$, and 1,020 $\mu \mathrm{S} / \mathrm{cm}$, respectively.

\section{PCP-spiked soils}

As mentioned earlier, field sand was used for this study to represent highly porous media and kaolin was used as low permeability soil. The properties of field sand and kaolin are shown in Table 2. The field sand had an organic content of $0.8 \%$, whereas the kaolin had no organic content. About $600 \mathrm{~mL}$ hexane was used to dissolve $100 \mathrm{mg}$ of solid PCP. To ensure all the PCP solids were dissolved, the PCP-hexane mixture was placed on a magnetic stirrer for about $45 \mathrm{~min}$. Then, $1 \mathrm{~kg}$ of soil was weighed in a large glass beaker. After mixing the soil-PCP-hexane mixture for $30 \mathrm{~min}$, the mixture was placed in a ventilation hood nearly 7 days for the mixture to dry. During the drying period the soil mixture was mixed regularly to ensure uniform drying.

\section{Reactivity experiments}

A series of batch experiments was conducted using kaolin and field sand soils spiked with PCP at 100 and $89 \mathrm{mg} / \mathrm{kg}$, respectively. Different NIP dosages $(4,10,20,50$, and $100 \mathrm{~g} / \mathrm{L})$ and different reaction times (1, 2, 4, 7, and 14 days) were

Table 2. Properties of Field Sand and Kaolin

\begin{tabular}{llc}
\hline Property & Field sand & Kaolin \\
\hline Mineralogy & Quartz & $\begin{array}{c}\text { Kaolinite: 100\%, } \\
\text { Muscovite: } \\
\text { trace, Illite: } \\
\text { trace }\end{array}$ \\
& & \\
Particle size distribution & & \\
$\quad$ (ASTM D 422): & & 0 \\
$\quad$ Gravel (\%) & 0.1 & 4 \\
Sand (\%) & 98.1 & 18 \\
Silt (\%) & 1.7 & 78 \\
$\quad$ Clay (\%) & 0.1 & 2.52 \\
Specific gravity (ASTM D 854) & 2.71 & $1.0 \times 10^{-8}$ \\
Hydraulic conductivity (cm/s) & $2.67 \times 10^{-3}$ & \\
$\quad$ (ASTM D 2434 & & 4.9 \\
$\quad$ and ASTM D 5084) & & $\sim 0$ \\
pH (ASTM 4972) & 7.5 & CL \\
Organic content (\%) & 0.8 & \\
$\quad$ (ASTM D 2974) & & \\
USCS classification & \multicolumn{2}{c}{ SP } \\
$\quad$ (ASTM D 2487) & & \\
\hline
\end{tabular}

applied to evaluate the effect of NIP dosage and time on PCP degradation in soils. The experiments were conducted in $50 \mathrm{~mL}$ bottles at a soil:solution mixing ratio of $1 \mathrm{~g}: 5 \mathrm{~mL}$. Thus, $25 \mathrm{~mL}$ NIP solution was added to $5 \mathrm{~g}$ of spiked soil. The samples were placed in a rotating tumbler for $1,2,4,7$, and 14 days. In preparing the bare-NIP solution, a certain amount of NIP slurry was weighed on the analytical balance and mixed with 1 liter of the groundwater solution and shaken well to ensure homogeneous mixing. Aluminum LM-NIP were prepared by the addition of solid aluminum lactate to a known weight of NIP slurry to achieve a $10 \%$ concentration $(w / w)$ of aluminum lactate; the mixture of NIP and aluminum lactate was then shaken with a vortex mixture for several minutes before addition to the groundwater solution. After the reaction period, the NIP-soil slurry was centrifuged at 7,000 rpm for $10 \mathrm{~min}$ to separate the solution from the soil. The supernatant solution and the soil were analyzed for PCP and phenol. All experiments were carried out in triplicates.

\section{Chemical analysis}

After separation of soil and solution, the soil was extracted with solvent for $24 \mathrm{~h}$ using 1:3 (v/v) mixture of water and ethanol (Khodadoust et al., 1999). The extracts were then analyzed for PCP using gas chromatography (GC) according to USEPA method 8041 (USEPA, 1996). The aqueous supernatant solutions were filtered and acidified with $\mathrm{HCl}$ to less than $\mathrm{pH} 2$ before liquid-liquid extraction into hexane followed by injection into GC. The extracts and supernatant solutions were also analyzed for phenol using GC according to USEPA method 8041.

\section{Results}

The degradation of PCP as a function of bare-NIP dosage is shown in Fig. 1a and b for field sand and kaolin, respectively. These results show that greater degradation of PCP occurred in $24 \mathrm{~h}$ with increasing dosage of bare-NIP from 4 to $100 \mathrm{~g} / \mathrm{L}$. The degradation in field sand increased from $87 \%$ for $4 \mathrm{~g} / \mathrm{L}$ NIP to $98 \%$ for with $100 \mathrm{~g} / \mathrm{L}$ NIP. The degradation in kaolin increased from $85 \%$ with $4 \mathrm{~g} / \mathrm{L}$ NIP to $99 \%$ with $100 \mathrm{~g} / \mathrm{L}$ NIP. For both field sand and kaolin, nearly complete degradation of PCP was obtained with $100 \mathrm{~g} / \mathrm{L}$ bare-NIP; similar results were obtained by Reddy and Karri (2008), who reported that higher NIP concentrations increased the removal of PCP from soil, where near $100 \%$ destruction of PCP in kaolin was obtained with $100 \mathrm{~g} / \mathrm{L}$ bare-NIP. The reactivity of bare-NIP with PCP was greater by $2 \%-3 \%$ for kaolin than for field sand at higher dosages of NIP. For both field sand and kaolin, there was a greater increase in reactivity from 4 to $20 \mathrm{~g} / \mathrm{L}$ dosage of NIP followed by a lesser increase in reactivity from 20 to $100 \mathrm{~g} / \mathrm{L}$ of bare-NIP. The $\mathrm{pH}$ values of the field sand and kaolin systems are also shown in Fig. 1a and b, respectively. The $\mathrm{pH}$ data show that the application of larger dosages of bare-NIP led to higher $\mathrm{pH}$ values for both field sand and kaolin. For field sand, the $\mathrm{pH}$ increased from 7.67 at $4 \mathrm{~g} / \mathrm{L} \mathrm{NIP}$ dosage to 8.84 at $100 \mathrm{~g} / \mathrm{L}$ NIP dosage. For kaolin, the $\mathrm{pH}$ increased from 8.19 at $4 \mathrm{~g} / \mathrm{L}$ NIP dosage to 9.59 at $100 \mathrm{~g} / \mathrm{L}$ NIP dosage. The $\mathrm{pH}$ of the kaolin system was greater than the $\mathrm{pH}$ of the field sand system.

To compare the reactivity of the smallest dosage of NIP with reaction time to the higher reactivity of the larger dosages of NIP in $24 \mathrm{~h}$, the reactivity of NIP with PCP was 

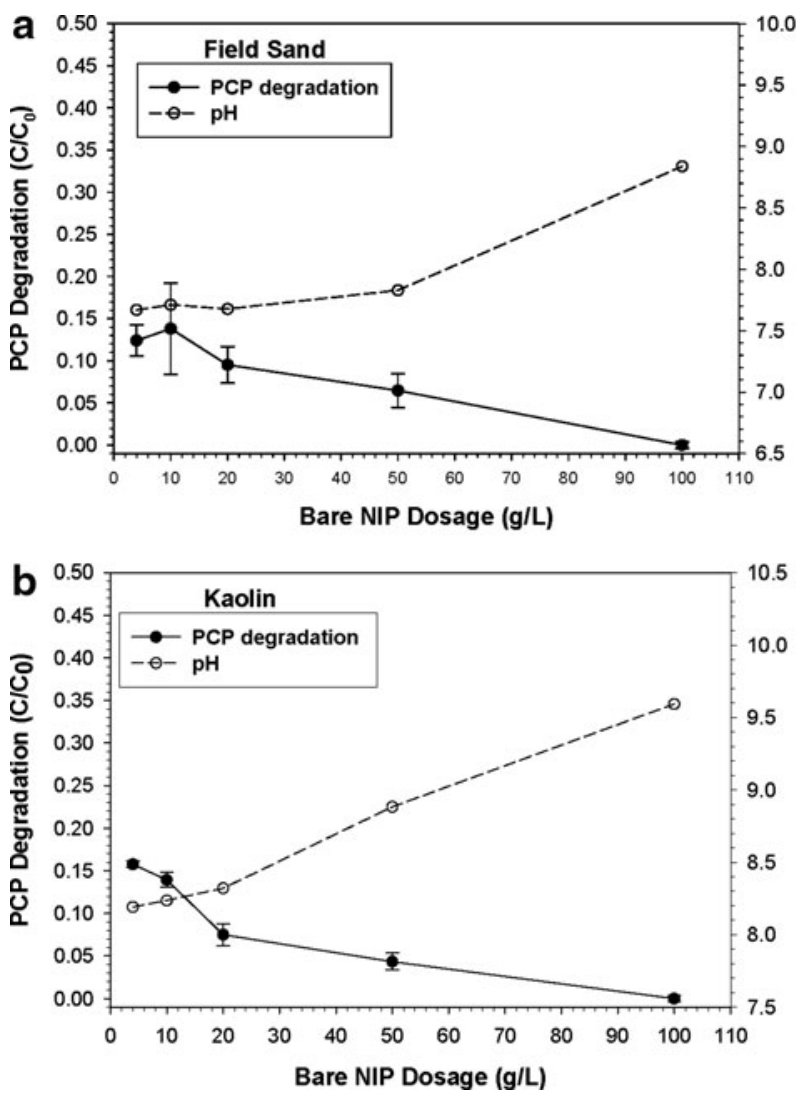

FIG. 1. Degradation of PCP as a function of bare-NIP dosage in (a) field sand and (b) kaolin. NIP, nanoscale iron particles; PCP, pentachlorophenol.

evaluated as a function of reaction time for up to 14 days using a bare-NIP dosage of $4 \mathrm{~g} / \mathrm{L}$. The same testing was performed using $4 \mathrm{~g} / \mathrm{L}$ LM-NIP. The results of reactivity of NIP with PCP as a function of time and surface modification are shown in Fig. $2 a$ and $b$ for field sand and kaolin, respectively. The PCP degradation data presented in Fig. $2 a$ and $b$ show that greater destruction of PCP in both soils was obtained with bare-NIP than with LM-NIP. The PCP degradation with LM-NIP after 1 day was less than the reactivity with bare-NIP, but the PCP degradation increased after 14 days for both field sand and kaolin. For field sand the degradation with LM-NIP was 79\% after 1 day but increased to $90 \%$ after 7 days, whereas the degradation with bare-NIP increased from $87 \%$ after 1 day to $93 \%$ after 7 days. Thus, the difference in reactivity between NIP and LM-NIP was larger initially but diminished after 7 days for the field sand systems. For kaolin, although the degradation with LM-NIP increased with time, it was appreciably less ( $10 \%$ to $12 \%$ ) than the degradation with bareNIP from 2 to 14 days of reaction. There was no significant increase in PCP degradation in field sand for both bare-NIP and LM-NIP systems from 4 to 14 days, whereas there was no significant increase in PCP degradation in kaolin for bare-NIP system from 7 to 14 days. Thus, the difference in reactivity between bare-NIP and LM-NIP did not diminish as much with time for kaolin.

The $\mathrm{pH}$ of the soil-NIP systems was measured as a function of time. The $\mathrm{pH}$ data are shown in Fig. 3a and $\mathrm{b}$ for field sand and kaolin, respectively. The $\mathrm{pH}$ data show that the $\mathrm{pH}$ values
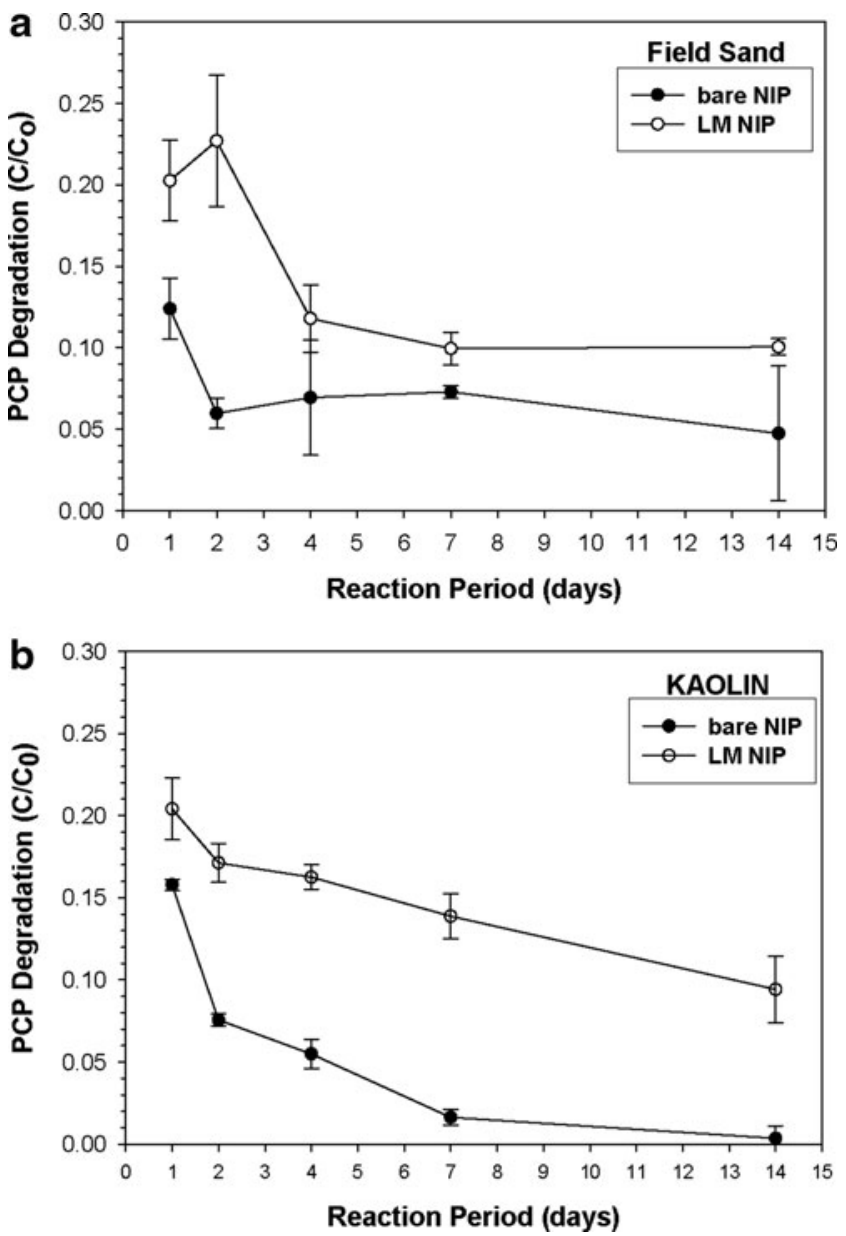

FIG. 2. Degradation of PCP as a function of reaction time using $4 \mathrm{~g} / \mathrm{L}$ bare-NIP and modified NIP in (a) field sand and (b) kaolin.

for the bare-NIP systems were higher than the $\mathrm{pH}$ values for the LM-NIP systems for both field sand and kaolin. The data also show that the difference in $\mathrm{pH}$ values between the bareNIP and LM-NIP systems were greater for kaolin than for field sand, where the bare-NIP-kaolin systems had the largest $\mathrm{pH}$ values $(>8)$ and the LM-NIP-kaolin systems the lowest $\mathrm{pH}$ values $(<6.5)$. For both field sand and kaolin systems with LM-NIP, the $\mathrm{pH}$ values increased between 1 and 4 days but remained relatively unchanged between 4 and 14 days. For field sand-bare-NIP system, the $\mathrm{pH}$ increased from 1 to 2 days, decreased from 2 to 4 days, and remained constant thereafter. For kaolin-bare NIP system, the $\mathrm{pH}$ decreased slightly from 1 to 2 days, and remained relatively unchanged thereafter. For all $\mathrm{pH}$ readings, the variation in $\mathrm{pH}$ of the triplicate samples was quite small; therefore, no error bars have been shown for $\mathrm{pH}$.

\section{Discussion}

\section{Effect of bare-NIP dosage on PCP degradation}

The data in Fig. 1 show that degradation of PCP increased with increasing bare-NIP dosage.

For bare-NIP dosages, the greater reactivity of NIP at higher NIP dosages was due to the presence of greater NIP 

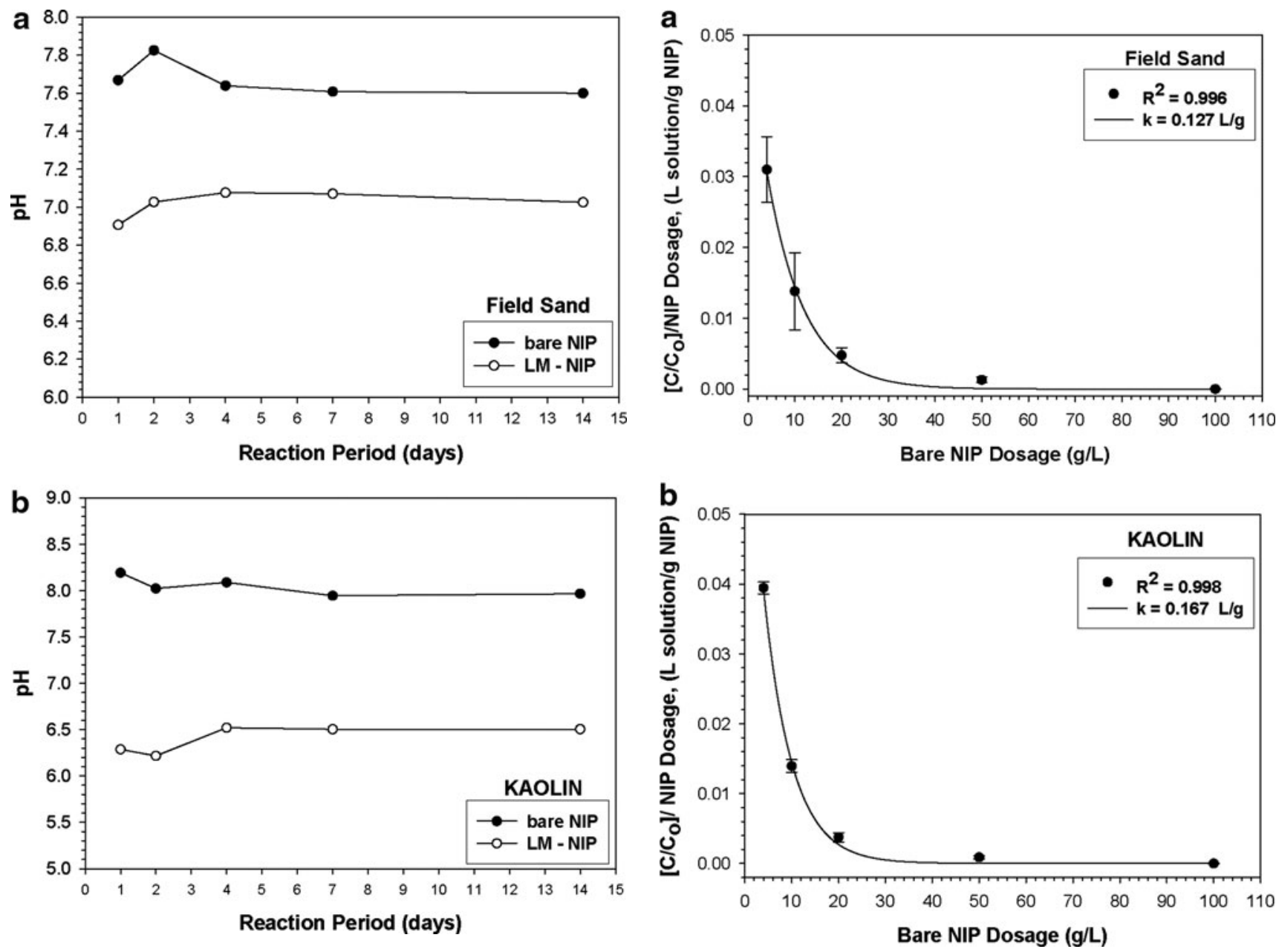

FIG. 3. $\mathrm{pH}$ as a function of reaction time using $4 \mathrm{~g} / \mathrm{L}$ bareNIP and modified NIP in (a) field sand and (b) kaolin.

surface area. The efficiency of PCP degradation relative to the dosage of bare-NIP depends on the available NIP reactive surface area. The degradation of PCP normalized to the dosage of NIP is shown as a function of NIP dosage in Fig. 4a and $\mathrm{b}$ for field sand and kaolin systems, respectively. The results presented in Fig. 4 show that the efficiency of NIP for PCP degradation decreased markedly for both soils with increasing dosage of NIP. The results indicate that for both field sand and kaolin, the rate of PCP degradation relative to NIP dosage declined appreciably from 4 to $20 \mathrm{~g} / \mathrm{L}$ NIP followed by slow decline between 20 and $100 \mathrm{~g} / \mathrm{L}$ NIP. The drastic decline between 4 and $20 \mathrm{~g} / \mathrm{L}$ NIP indicates that PCP degradation was most efficient at $4 \mathrm{~g} / \mathrm{L}$ NIP dosage among the applied NIP dosages. The slow decline between 20 and $100 \mathrm{~g} / \mathrm{L}$ NIP indicates that the application of NIP above the $20 \mathrm{~g} / \mathrm{L}$ dosage is inefficient. The nonlinear regression of data using a first-order exponential decay equation was carried out as follows:

$$
\left\{\left(C / C_{0}\right) / D\right\}=\left\{\left(C / C_{0}\right) / D\right\}_{\max }\left[e-{ }^{k e f f} D\right]
$$

where $D(\mathrm{~g} / \mathrm{L})$ and keff $(\mathrm{L} / \mathrm{g})$ were the bare-NIP dosage and the degradation efficiency coefficient, respectively. The $k_{\text {eff }}$ values were $0.1268 \pm 0.0089$ and $0.1667 \pm 0.0090 \mathrm{~L} / \mathrm{g}$ for field sand and kaolin, respectively, and the $\left\{\left(C / C_{0}\right) / D\right\}_{\max }$ values

FIG. 4. NIP dosage normalized degradation of PCP in (a) field sand and (b) kaolin.

were 0.0512 and 0.0766 for field sand and kaolin, respectively. The results indicate that the decrease in efficiency of PCP degradation relative to bare-NIP dosage was greater for kaolin than for field sand.

\section{Effect of $\mathrm{pH}$ on PCP degradation}

For equivalent mass of bare-NIP (equivalent bare-NIP surface area), the data shown in Fig. 1 indicate that PCP degradation was greater for kaolin than for field sand. The effect of $\mathrm{pH}$ on reactivity is another factor in addition to the availability of greater reactive surface (sites) of NIP at higher NIP dosages. For an equivalent dosage of bare-NIP in field sand and kaolin systems, the PCP degradation was affected by the $\mathrm{pH}$ of the soil-NIP systems. PCP is an ionizable organic compound with a $\mathrm{p} K_{\mathrm{a}}$ value of 4.7 that dissociates with respect to $\mathrm{pH}$; hence, the desorption and the subsequent degradation of PCP were affected by $\mathrm{pH}$. Greater desorption of PCP would lead to greater degradation. The bare-NIP solution was very alkaline with a $\mathrm{pH}$ of 10.7 . The $\mathrm{pH}$ of the NIPkaolin systems was higher than the $\mathrm{pH}$ of the NIP-field sand systems, whereas the $\mathrm{pH}$ of the kaolin soil was acidic $(\mathrm{pH} 4.9)$ and lower than the $\mathrm{pH}$ of the field sand, which was neutralalkaline ( $\mathrm{pH} 7.5$ ) as shown in Table 2. The higher $\mathrm{pH}$ observed 
in NIP-kaolin systems was due to the low buffering capacity of the kaolin, which upon contact with the alkaline bare-NIP solution increased to $\mathrm{pH}$ values between 8.19 and 9.59, whereas the $\mathrm{pH}$ of NIP-field sand systems ranged from 7.67 to 8.84 (versus a field sand $\mathrm{pH}$ of 7.5). The higher $\mathrm{pH}$ of the NIPkaolin systems would be conducive to a greater desorption of PCP from kaolin.

The PCP degradation data in Fig. $2 a$ and $b$ and the $p H$ values presented in Fig. $3 a$ and $b$ show that greater degradation occurred for systems with higher $\mathrm{pH}$ values; more degradation was observed for bare-NIP-kaolin system (higher $\mathrm{pH}$ ) than for the bare NIP-field sand system (lower $\mathrm{pH})$, whereas more degradation was observed for LM-NIPfield sand system (higher $\mathrm{pH}$ ) than for LM-NIP-kaolin system (lower $\mathrm{pH}$ ). The $\mathrm{pH}$ of the bare-NIP solution was high (10.7), whereas the $\mathrm{pH}$ of the LM-NIP slurry was much lower due to the acidity of aluminum lactate. Two factors may have contributed to the effect of $\mathrm{pH}$ on the reactivity of NIP with $\mathrm{PCP}$, whereas the lower $\mathrm{pH}$ setting increased the corrosion rate of NIP; thus, generating more electrons for reductive dechlorination of $\mathrm{PCP}$, the desorption, and subsequent degradation of $\mathrm{PCP}$ were favored at higher $\mathrm{pH}$ values, as evidenced by the greater degradation of PCP with bare-NIP in kaolin (higher $\mathrm{pH}$ ) rather than in field sand (lower $\mathrm{pH}$ ). The difference in reactivity between bare-NIP and LM-NIP diminished after 7 days for field sand, whereas the difference in reactivity between bare-NIP and LM-NIP did not diminish with time for kaolin. The smaller difference in $\mathrm{pH}$ values of the bare-NIP and LM-NIP systems for field sand (7.6 vs. 7.0) and the larger difference in $\mathrm{pH}$ values of the bare-NIP and LM-NIP systems for kaolin (8.0 vs. 6.5) may have attributed to the smaller and the large differences in PCP degradation with time for the field sand and kaolin systems, respectively.

\section{Effect of dispersant and reaction period on PCP degradation}

The PCP degradation data presented in Fig. 2a and $b$ for field sand and kaolin, respectively, show that PCP degradation in general increased with time. The degradation in field sand did not increase appreciably from 4 to 14 days with bareNIP and LM-NIP, whereas the degradation in kaolin continued to increase from 4 to 7 days with bare-NIP and continued to increase from 7 to 14 days with LM-NIP. The continued degradation of PCP in the kaolin/LM-NIP system indicate that degradation was hindered by the surface desorption of $\mathrm{PCP}$ from kaolin at lower $\mathrm{pH}$. The longer contact time allowed for the continued desorption and degradation of PCP in the kaolin/LM-NIP system.

The oxidation-reduction potential (ORP) for both NIP and LM-NIP was measured for NIP-field sand systems with reaction period. The ORP for the bare-NIP systems was about $-480 \mathrm{mV}$ for 1 day and increased to $+200 \mathrm{mV}$ within 4 days, whereas the ORP for the LM-NIP systems was about $-500 \mathrm{mV}$ after 1 day and increased to about $+100 \mathrm{mV}$ after 4 days. Both systems were very reducing for the first day but became much less reducing within 2 days. This increase in ORP may have contributed to the decrease in reaction rate after 2 days in field soil systems. The ORP of the LM-NIP system was lower $(100 \mathrm{mV})$ than that of bare-NIP system $(200 \mathrm{mV})$ for the period from 4 to 14 days, indicating that the presence of aluminum lactate in the NIP solution led to moderately greater reducing conditions after the initial rapid reaction period of the first 2 days. The coating of the NIP surface with aluminum lactate also hindered the reactivity of LM-NIP despite the lower $\mathrm{pH}$ setting in the presence of aluminum lactate. Other authors have reported similar trends whereby the addition of dispersant decreased the rate of reactivity (Saleh et al., 2007; Yang et al., 2007). Tratnyek et al. (2001) reported that the reduction of nitrobenzene and trichloroethylene was subdued by the presence of field organic matter.

\section{Phenol production and PCP dechlorination}

The degradation of PCP occurred via reductive dechlorination (Khodadoust et al., 1997), where the higher chlorinated compound is reduced to lower chlorinated compounds. For PCP (penta-CP), the general dechlorination pathway is as follows:

$$
\begin{aligned}
& \text { pentaCP } \rightarrow \text { tetra-CPs } \rightarrow \text { tri-CPs } \rightarrow \text { di-CPs } \rightarrow \\
& \text { mono-CPs } \rightarrow \text { phenol }
\end{aligned}
$$

In our study, the production of phenol was monitored with time to determine the dechlorination activity for bare-NIP and LM-NIP systems for field sand and kaolin. While phenol was detected in all samples, no tetra-CPs and tri-CPs were detected. The production of phenol per mole of PCP degraded as a function of time is shown in Fig. $5 a$ and $b$ for field sand and kaolin systems, respectively, using $4 \mathrm{~g} / \mathrm{L}$ NIP. The phenol production data presented in Fig. 5a and b show that nearly complete dechlorination of PCP occurred in both the field sand and kaolin systems after 14 days, where the ratio (mole phenol/mole PCP) becomes nearly 1.0. The data also indicate that the nearly complete dechlorination of PCP occurred faster in the field sand systems (within 4 days) than in the kaolin systems (within 14 days).

The data were fitted to a first-order rate equation for the increase in the production of phenol with time:

$$
\left[C_{\text {phenol }} / C_{\mathrm{PCP}}\right](t)=\left[C_{\text {phenol }} / C_{\mathrm{PCP}}\right]_{\max }\left(1-e^{-k t}\right),
$$

where $\left[C_{\text {phenol }} / C_{\mathrm{PCP}}\right]$ is the mole of phenol produced per mole of PCP degraded and $k$ is the first-order rate constant. The rate constants were $0.792 \pm 0.032$ and $1.138 \pm 0.119 \mathrm{~d}^{-1}$ for bare-NIP and LM-NIP in field sand systems, respectively, and $0.402 \pm 0.084 \mathrm{~d}^{-1}$ for bare-NIP in kaolin system. At a $95 \%$ confidence interval, comparison of the rate constants for field sand systems resulted in a $p$-value of $0.021(<0.05)$, indicating that the rate of phenol production was significantly faster for LM-NIP than for bare-NIP in field sand systems. Similarly, at a 95\% confidence interval, comparison of the rate constants for kaolin and field sand systems with bare-NIP resulted in $p=0.006$, indicating that the rate of phenol production was significantly faster in field sand than in kaolin.

A possible factor for the more rapid dechlorination in field sand systems would be the faster desorption of PCP, which would have led to the faster reaction of desorbed PCP in the aqueous phase with the surface of NIP in field sand systems, indicating that the reaction of dissolved PCP with NIP was possibly more mass transfer limited in the kaolin systems than in the field sand systems. Kaolin clay particles have a much larger surface area with smaller particle size, whereas sand 
a

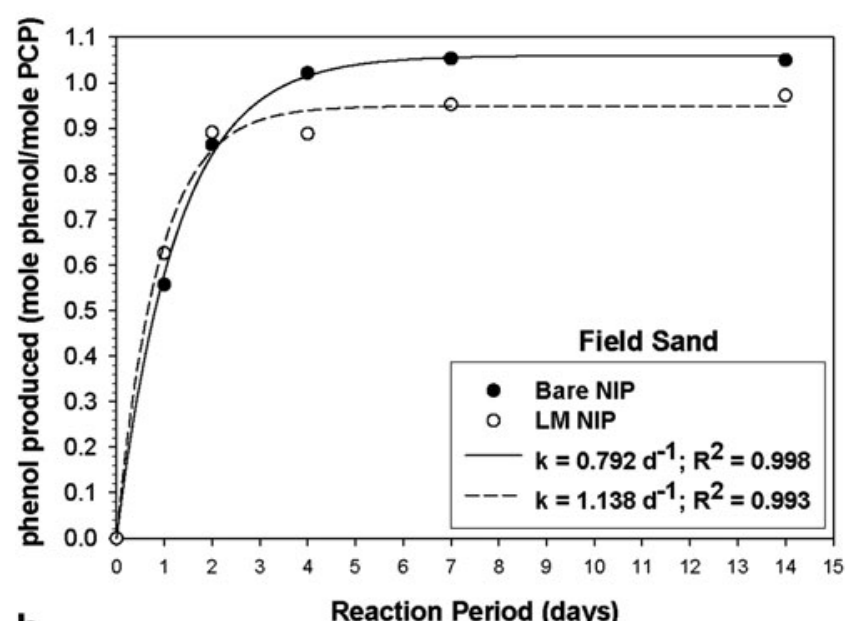

b

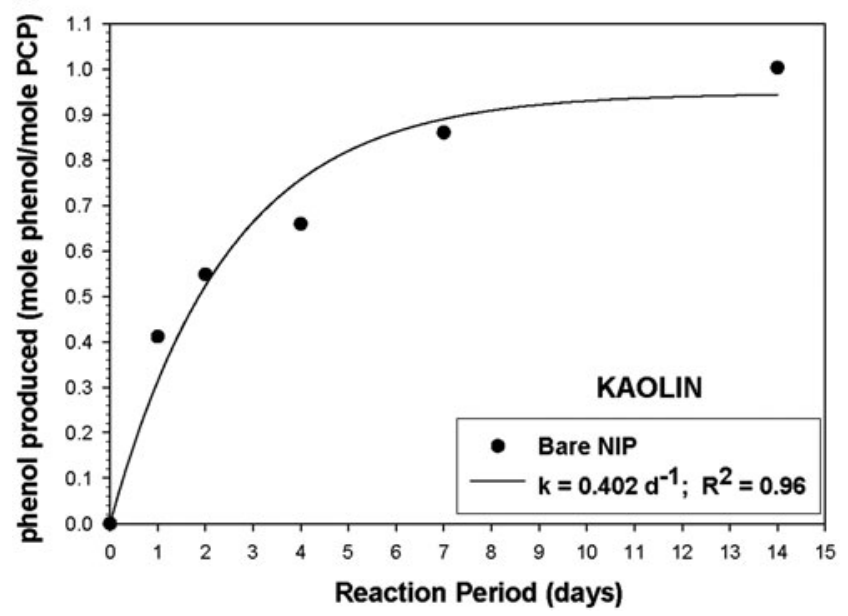

FIG. 5. Production of phenol and PCP dechlorination rate using $4 \mathrm{~g} / \mathrm{L}$ NIP in (a) field sand and (b) kaolin.
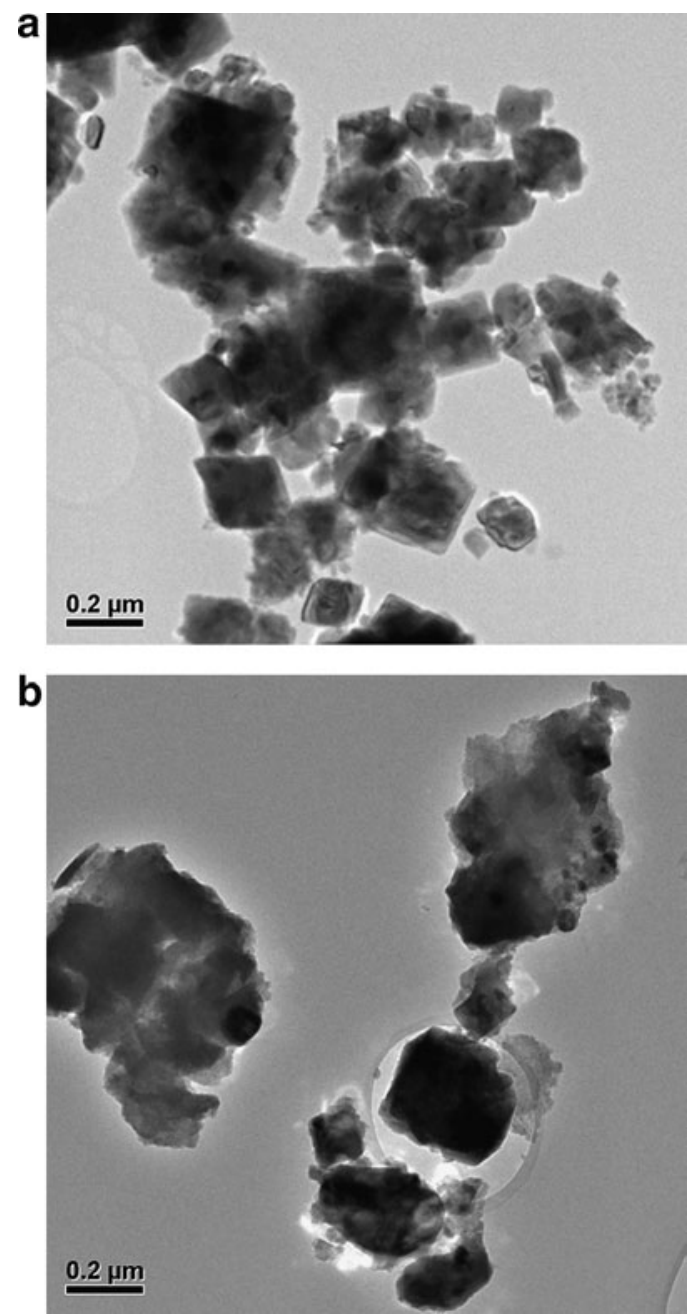

FIG. 6. Transmission electron microscopy images for (a) bare-NIP and (b) lactate-modified NIP.

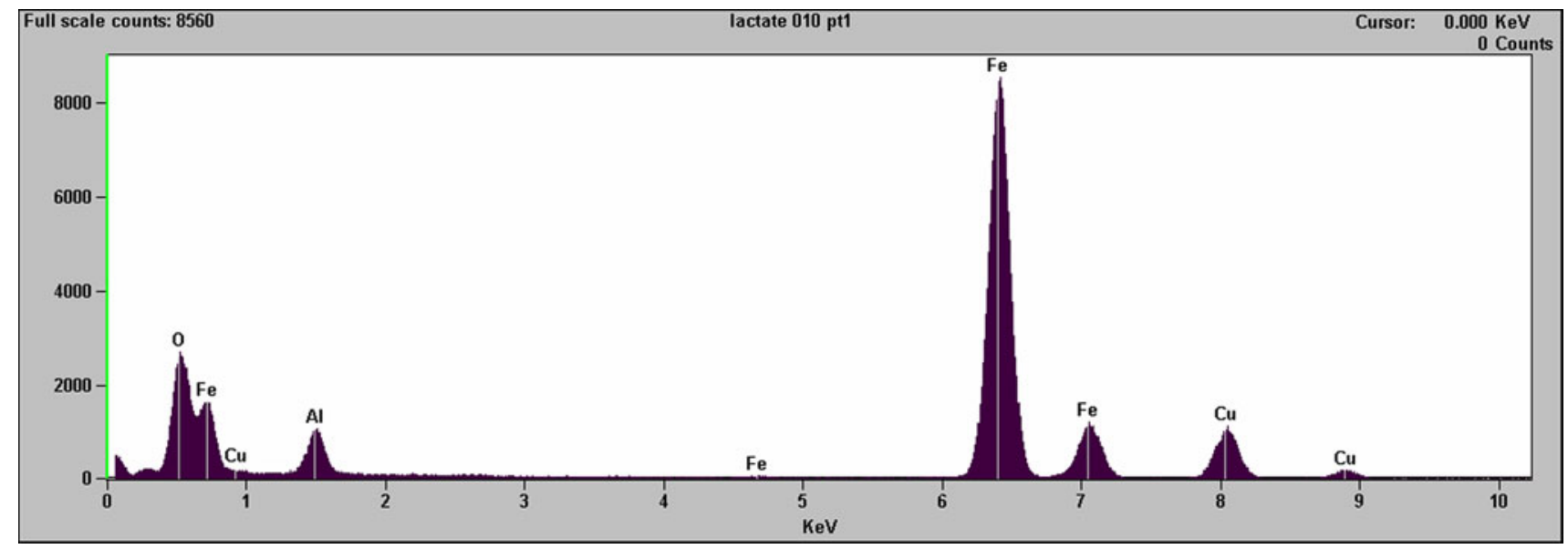

FIG. 7. Transmission electron microscopy/X-ray electron diffraction system elemental analysis of lactate-modified NIP. 

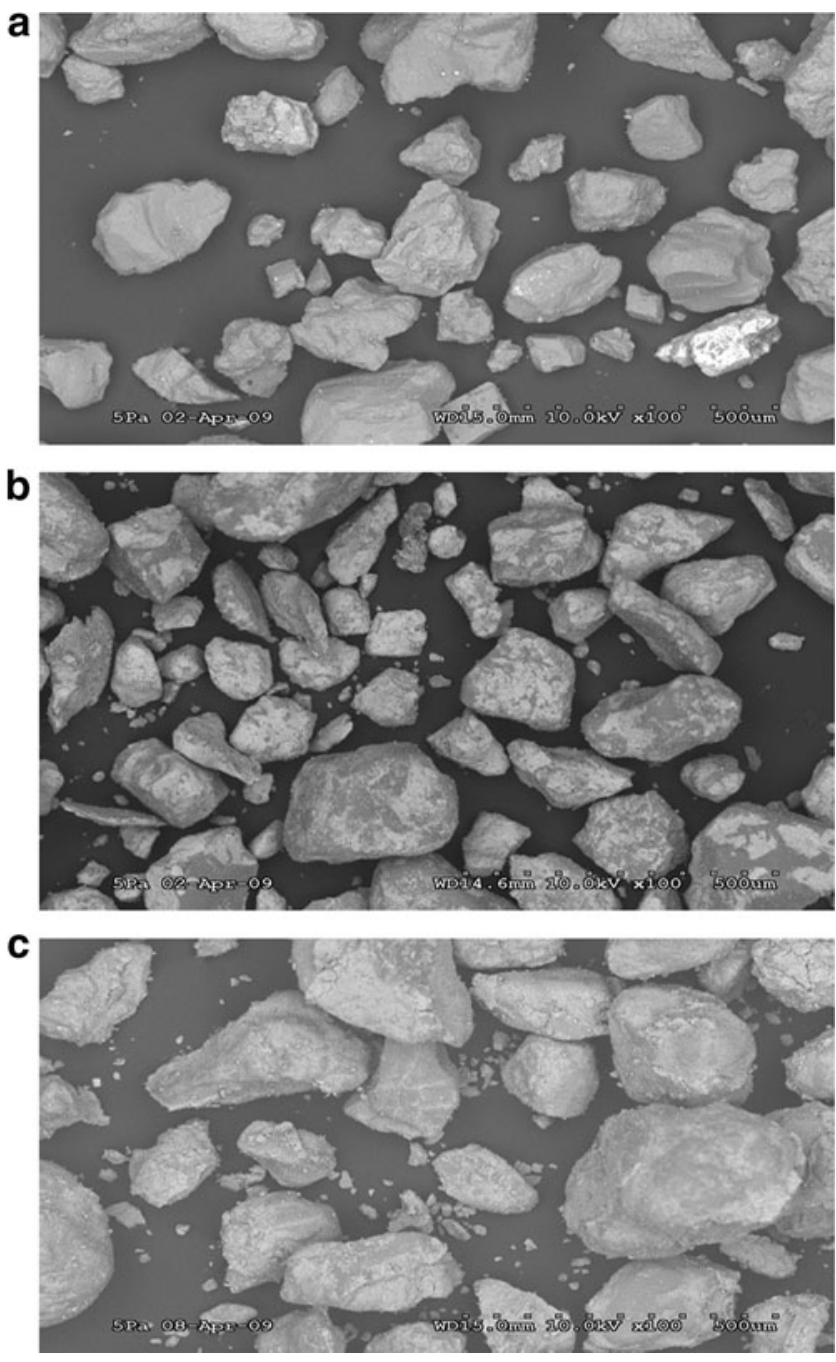

FIG. 8. Scanning electron microscopy micrographs for field sand (a) without NIP, (b) with bare-NIP, and (c) with modified NIP.

particles have larger particle size with less surface area. Although we hypothesize that a more rapid desorption of PCP from field sand would have been conducive to the more rapid dechlorination of PCP in field sand systems, further study on sorption kinetics is required to explain the difference between the field sand and kaolin systems.

\section{Surface analysis}

The surface analysis of bare-NIP and LM-NIP systems was carried out using electron microscopy. Transmission electron microscopy (TEM) analysis was conducted to investigate the differences in the physical and chemical characteristics of the bare-NIP and the LM-NIP. The representative TEM images of bare-NIP and LM-NIP are shown in Fig. 6a and b. The TEM analysis shows that the bare-NIP particles appear facetted with particle size ranging from 100 to $300 \mathrm{~nm}$. X-ray Electron Diffraction System (XEDS) shows that the bare-NIP sample contains not just iron and oxygen, but also minute concentrations of aluminum. The micrograph from the LM-NIP sample shows that some of the NIP particles (facetted) are bounded by a blurry, featureless medium. The elemental analysis obtained from XEDS for the LM-NIP is shown in Fig. 7. XEDS on this medium revealed high concentration of aluminum; electron diffraction indicates that it was amorphous, which seems to suggest that this blurry medium was likely to be aluminum lactate. The amorphous nature of the aluminum lactate might allow for the penetration of the target organic compound into the LM-NIP matrix and subsequent reaction on the surface of NIP.

Scanning electron microscopy (SEM) analysis was carried out for field sand-NIP systems. The SEM micrographs are shown in Fig. 8a-c for field sand particles without NIP, coated with bare-NIP, and coated with LM-NIP, respectively. The SEM micrographs shown in Fig. 8 indicate a distribution of bare-NIP on the surface of the soil particles, whereas there was a more uniform distribution of LM-NIP on the surface of the sand particles. Elemental analysis carried out by XEDS on all soil samples indicate the presence of iron on the surface of soil samples with bare-NIP and LM-NIP. Although XEDS indicates the presence of aluminum on the sand surface for both bare-NIP and LM-NIP, there was much more aluminum on the surface of samples coated with LM-NIP. The aluminum-to-iron mass ratio for LM-NIP samples was several times greater than the same ratio for bare-NIP samples due to the application of $10 \%$ aluminum lactate in the LM-NIP slurry. This means that an appreciable proportion of aluminum lactate remained on the NIP surface after contact between the LM-NIP and the field sand.

\section{Conclusions}

The LM-NIP have the potential to transform PCP in soils and groundwater. Degradation of PCP was achieved at lower bare-NIP dosages, but nearly complete destruction was obtained at the highest dosage of $100 \mathrm{~g} / \mathrm{L}$ bare-NIP. The results indicate that reactivity increased with increasing NIP dosage. At lowest dosage of $4 \mathrm{~g} / \mathrm{L} \mathrm{NIP}$, higher degradation was observed with increasing reaction time. The LM-NIP was effective for the degradation of PCP in both field sand and kaolin. Although the reactivity was less than that of bare-NIP, the LM-NIP became as effective as bare-NIP with time. Nearly complete dechlorination of PCP was obtained for both field sand and kaolin with bare-NIP.

\section{Acknowledgment}

Financial support for this project was provided by the U.S. National Science Foundation (Grant CMMI \#0727569), which is gratefully acknowledged.

\section{Author Disclosure Statement}

No competing financial interests exist.

\section{References}

Cameselle, C., Darko-Kagya, K., Khodadoust, A., and Reddy, K.R. (2008). Influence of type and concentration of dispersants on the zeta potential of reactive nanoiron particles. In Proceedings of the International Environmental Nanotechnology Conference. USEPA, Chicago.

Cheng, R., Wang, J., and Zhang, W. (2006). Comparison of reductive dechlorination of $p$-chlorophenol using $\mathrm{Fe} 0$ and nanosized $\mathrm{Fe}^{0}$. J. Hazard. Mater. 144, 334. 
Cheng, R., Wang, J., and Zhang, W. (2008). Degradation of chlorinated phenols by nanoscale zero-valent iron. Environ. Sci. Eng. China 2, 103.

Choe, S., Lee, S., Chang, Y., Hwang, K., and Khim, J. (2001). Rapid reductive destruction of hazardous organic compounds by nanoscale $\mathrm{Fe}^{0}$. Chemosphere 42,367 .

Kanel, S.R., and Choi, H. (2007). Transport characteristics of surface-modified nanoscale zero-valent iron in porous media. Water Sci. Technol. 55, 157.

Keither, L.H., and Telliard, W.A., (1979). Priority pollutants: I-a perspective view. Environ. Sci. Technol. 13, 416.

Khodadoust, A.P., Suidan, M.T., Acheson, C.M., and Brenner, R.C. (1999). Solvent extraction of pentachlorophenol from contaminated soils using water-ethanol mixtures. Chemosphere 38, 2681.

Khodadoust, A.P., Wagner, J.A., Suidan, M.T., and Brenner, R.C. (1997). Anaerobic treatment of PCP in fluidized-bed GAC bioreactors. Water Res. 31, 1776.

Kim, Y.H., and Carraway, E.R. (2000). Dechlorination of pentachlorophenol by zerovalent iron and modified zerovalent irons. Environ. Sci. Technol. 34, 2014.

Liu, Y., Majetich, S.A., Tilton, R.D., Sholl, D.S., and Lowry, G.V. (2005). TCE dechlorination rates, pathways, and efficiency of nanoscale iron particles with different properties. Environ. Sci. Technol. 39, 1338.

Morales, J., Hutcheson, R., and Cheng, I.F. (2002). Dechlorination of chlorinated phenols by catalyzed and uncatalyzed $\mathrm{Fe}(0)$ and $\mathrm{Mg}(0)$ particles. J. Hazard. Mater. 90, 97.

Okinaka, K., Jazdanian, A.D., Dahmani, A.N., Nakano, J., Okita, T., and Kakuya, K. (2005). Degradation of trichloroethene with reactive nanoscale iron particles in simulated groundwater. In Proceedings of Environmental Nanotechnology. Symposia Papers presented before the Division of Environmental Chemistry American Chemical Society, Washington, DC.

Reddy, K.R., and Karri, M.R. (2008). Removal and degradation of pentachlorophenol in clayey soil using nanoscale iron par- ticles. Geotechnics of Waste Management and Remediation, Geotechnical Special Publication. New Orleans, LA. 177, 463.

Reddy, K.R., Khodadoust, A.P., and Darko-Kagya, K. (2008). Transport and reactivity of lactate-modified nanoscale iron particles in PCP-contaminated field sand. In Proceedings of the International Environmental Nanotechnology Conference. USEPA, Chicago, p. 261.

Saleh, N., Sirk, K., Liu, Y., Phenrat, T., Dufour, B., Matyjaszewski, K., Tilton, R.D., and Lowry, G.V. (2007). Surface modifications enhance nanoiron transport and NAPL targeting in saturated porous media. Environ. Eng. Sci. 24, 45.

Subramanian, B., Namboodiri, V., Khodadoust, A.P., and Dionysiou, D.D. (2010). Extraction of pentachlorophenol from soils using environmentally benign lactic acid solutions. J. Hazard. Mater. 174, 263.

Tratnyek, P.G., and Johnson, A.L. (2006). Nanotechnology for environmental cleanup. Nanotoday. 1, 44.

Tratnyek, P.G., Scherer, M.M., Deng, B., and Hu, S. (2001). Effects of natural organic matter, anthropogenic surfactants, and model quinones on the reduction of contaminants by zerovalent iron. Water Res. 35, 4435.

United States Environmental Protection Agency (USEPA). (1996). Test Methods for Evaluating Solid Wastes. EPA Method SW 846, 3rd edition, Washington, DC: U.S. Government Printing Office.

Varanasi, P., Fullana, A., and Sidhu, S. (2007). Remediation of PCB contaminated soils using iron nano-particles. Chemosphere 66, 1031.

Wang, C., and Zhang, W. (1997). Synthesizing nanoscale iron particles for rapid and complete dechlorination of TCE and PCBs. Environ. Sci. Technol. 31, 2154.

Yang, G.C.C., and Lee, H. (2005). Chemical reduction of nitrate by nanosized iron: kinetics and Pathways. Water Res. 39, 884.

Yang, G.C.C., Tu, H., and Hung, C. (2007). Stability of nanoiron slurries and their transport in the subsurface environment. Sep. Purif. Technol. 58, 166. 
